\title{
Femto-second laser applications in energy materials characterization
}

\author{
Robin White ${ }^{1}$, Tobias Volkenandt ${ }^{2}$, Stephen Kelly ${ }^{1}$ and Benjamin Tordoff ${ }^{2}$ \\ ${ }^{1}$ Carl Zeiss Microscopy GmbH, United States, ${ }^{2}$ Carl Zeiss Microscopy GmbH, Germany
}

In recent times, the effects of climate change from the prevailing impact of global warming are beginning to be seen at an ever-growing rate. Record high temperatures are measured around the globe year after year and we are now seeing evidence of their impact in various other climate catastrophes including fires, flooding, and unexpected winter storms resulting in a huge economic and humanitarian impact. To curve the global climate back to equilibrium, the amount of green house gases and other emissions must be reduced significantly. As such, new government policies and economic pressures are forcing a rapid shift toward alternative energy sources such as hydrogen, solar, wind, tidal, and nuclear; and energy storage and conversion devices such as fuel cells and batteries. As these technologies are required to meet operational demands, a massive research effort has been put forward within the scientific community to improve upon existing technology performance, as evidenced by the number of new innovations in architecture and applications. One important method in the innovation pipeline is microscopy - whereby the exact physical structure, interactions, and processes can be observed and the subsequent fundamental understanding used to design better materials.

A unique form of microscopy is X-ray Microscopy (XRM). Due to the non-destructive 3D imaging capabilities, this imaging methodology provides unique insight into material properties with comparatively minimal sample processing. With an imaging field of view ranging from tens of millimeters down to hundreds of micrometers, and resolution capabilities reaching $500 \mathrm{~nm}$ in laboratory instruments such as ZEISS Xradia Versa, large, high resolution, non-destructive, 3D volumes can be acquired. As many of these devices rely on materials and structures across a broad range of length scales to achieve their functionality, higher resolution imaging to form a multi-scale understanding of the material properties is essential. To provide further insight into sample properties to be used for analysis or modelling, other imaging systems can be utilized in a massively multi-scale correlative workflow. For many of these methods, such as X-ray nanotomography or analytical measurements such as SEM with EDS or EBSD, and atom probe tomography, sample preparation is required to access deeply buried regions of interest or to form suitable sample dimensions. Moving from large sample volumes to targeted, small, buried regions can be impractical utilizing traditional ion milling technologies, limiting the correlative capabilities. Mechanical preparation methods further risk disturbing the underlying microstructure during preparation and complicate correlative workflows. With their extremely rapid milling rates (orders of magnitude faster than traditional ion beam approaches) and minimal heat affected zone (HAZ), the fs-laser has brought about a revolution in advanced materials characterization capabilities. Recently, a fs-laser mill has been integrated into a commercial focused ion beam and scanning electron microscope (FIB-SEM) instrument, enabling numerous new capabilities, including targeted correlative workflows between XRM and high-resolution analytical imaging. 
We present here results combining X-ray tomography systems and other microscopy techniques into a correlative, multi-length scale analysis workflow using specifically designed hardware and software tools. We demonstrate several applications using this combined approach to enable targeted high-resolution imaging and analysis with applications toward batteries, fuel cells and nuclear materials.

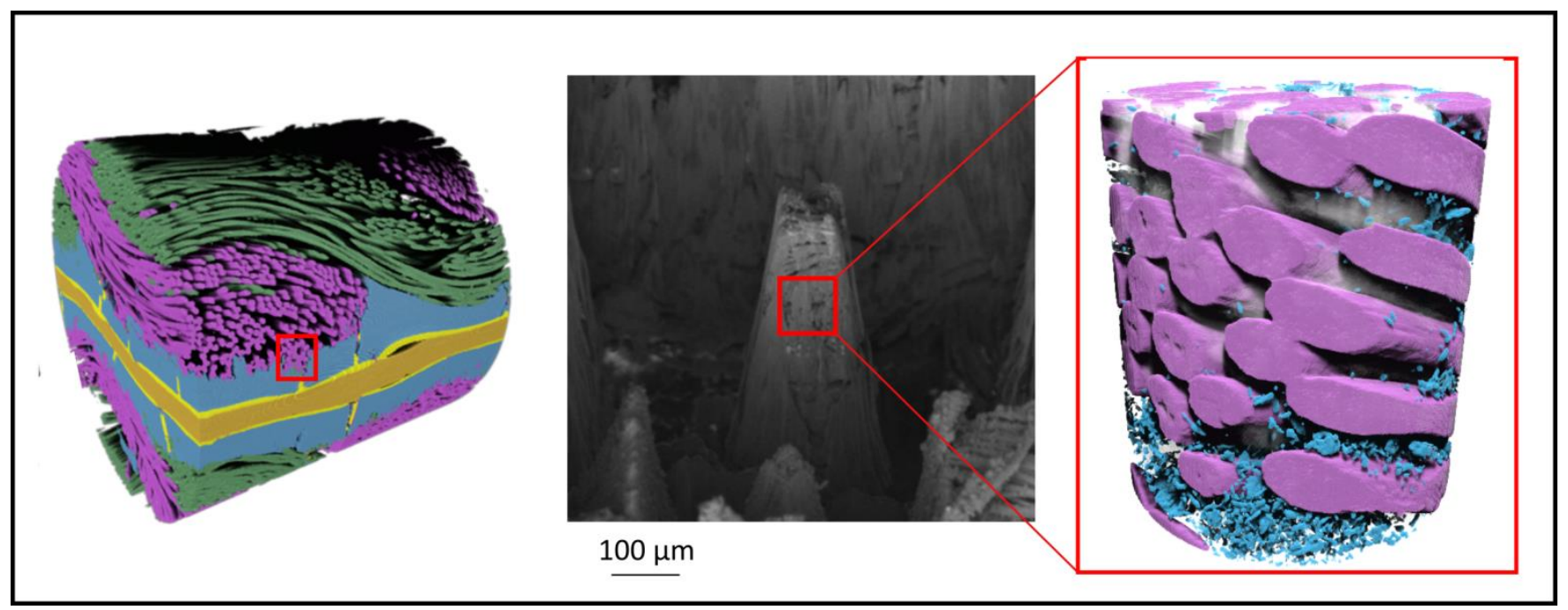

Figure 1. OVERVIEW OF LASER ABLATION TO CREATE A PILLAR FOR TARGETED HIGHRESOLUTION IMAGING OF POLYMER ELECTROLYTE FUEL CELL. LEFT: ZEISS XRADIA VERSA OVERVIEW SCAN. MID: LASER ABLATION TO ALLOW TARGETED HIGH RESOLUTION 3-DIMENSIONAL IMAGING BY FORMING PILLAR AFTER REMOVING LARGE VOLUME OF SAMPLE MATERIAL. RIGHT: 3D RENDERING OF THE PILLAR IMAGED WITH 64 NM / PIXEL USING THE ZEISS XRADIA ULTRA AT BURIED INTERFACE BETWEEN FIBERS AND MICROPOROUS LAYER. 